On the ninth day, the CSF contained a protein level of $133 \mathrm{mg} / \mathrm{dl}$ and one $1 \mathrm{ym}$ phocyte $/ \mathrm{mm}^{3}$. Electrophysiological study was performed on the twenty first hospital day. Motor nerve conduction of median nerve was $34 \mathrm{~ms}^{-1}$ with a distal motor latency of $4.5 \mathrm{~ms}$ (normal, up to $4 \cdot 2$ ). $\mathrm{F}$ responses were not obtained. Distal sensory nerve conduction velocity of median nerve was $29 \mathrm{~m}^{-1}$, sensory action potential being small $(1.5 \mu \mathrm{V})$. The extensor digitorum brevis muscle was denervated and motor conduction along the peroneal nerve could not be measured. Motor latency from the capitulum fibulae to the tibialis anterior was slightly prolonged, but the $\mathrm{M}$-wave was very decreased $(0.1 \mathrm{mV})$. Electromyography of the deltoid, abductor pollicis brevis, rectus femoris and tibialis anterior muscles revealed a pattern of discrete activity with an increase in mean duration of individual motor unit potentials. Fibrillation potentials and positive waves were only observed in the deltoid muscle. Six months after the onset of illness the patient still had pronounced proximal (MRC 2 to 3 ) and distal (MRC 3 to 4) weakness.

The diagnosis of ITP was strongly supported in our patient by the reduced number of circulating platelets, increased number of megakaryocytes, absence of splenomegaly and the response to prednisone. Specific IgG antibodies binding to defined platelet membrane glycoprotein antigens have been demonstrated in patients with chronic ITP, confirming the autoimmune nature of this disease. ' In acute ITP following a viral infection, the thrombocytopenic factors, be it specific platelet IgM antibodies or immune complexes, have not been defined. Failure to detect antibodies on platelets and in the serum of our patient might be the result of limited sensitivity of the immunofluorescence test. $^{1}$

This patient also fulfilled the diagnostic criteria for GBS. ${ }^{2}$ This is an inflammatory demyelinating neuropathy in which both protein and lipid antigens in peripheral nerve myelin are the target of immune attack, but the pathogenesis is still debatable. Experimental allergic neuritis induced by immunisation with the P2 protein has been considered a disease of cellular immunity and the experimental model of GBS. ${ }^{2}$ Alternatively, during acute-phase illness the serum of patients with GBS contains complementfixing antiperipheral nerve IgM antibodies. These antibodies bind to a neutral glycolipid in the myelin that has yet to be completely identified. ${ }^{3}$ Circulating immune complexes may constitute an additional type of pathogenetic mechanism to produce demyelination.

GBS rarely occurs concurrently with another autoimmune disorder. ${ }^{2}$ Coexistence of antibodies to factor VIII and peripheral nerve myelin has been demonstrated in a patient with chronic inflammatory polyradiculoneuropathy complicated by a coagulation disorder. ${ }^{4}$ The present association of GBS with a bleeding disorder due to ITP, as described in our case, has only been reported once previously. ${ }^{5}$

O COMBARROS J CALLEJA

L HERNÁNDEZ JM POLO Services of Neurology and Clinical Neurophysiology,

University Hospital "Marqués de Valdecilla",
Santander, Spain

Correspondence to: Dr Berciano.
1 Karpatkin S. Autoimmune thrombocytopenic purpura. Semin Hematol 1985;22:260-88.

2 Hughes RAC, Winer JB. Guillain-Barré syndrome. In: Matthews WB, Glaser G, eds. Recent advances in clinical neurology, Vol 4. Oxford: Blackwell, 1984:19-49.

3 Koski CL, Chou DKH, Jungalwala FB. Antiperipheral nerve myelin antibodies in Guillain-Barre syndrome bind a neutra glycolipid of peripheral myelin and cross1989;84:280-7.

4 Berger JR, Rosenfeld WE, Sheremata WA, et al. Chronic inflammatory polyradiculoneuropathy complicated by factor VIII antibody. Neurology 1983;33:1224-6.

5 Gross PT. Acute idiopathic polyneuritis and idiopathic thrombocytopenic purpura. JAMA 1980;243:256-7.

Isolated cerebellar syndrome: atypical form of cerebral malaria

The neurological manifestations of malaria are usually associated with the febrile attack, their outstanding features being the seizures and an impaired state of consciousness. We report a case of isolated cerebellar syndrome, a more benign complication of malaria, not related to the febrile attack.

A 31 year old French man, with no past medical history was admitted on 21 March 1989 at another hospital for a rapidly progressive ataxia. Four months previously, the patient worked as a member of the French Cooperation Group in Burkina Faso, and did not take regular prophylactic anti-malaria drugs. In January 1989, the patient had an attack of fever $\left(40^{\circ} \mathrm{C}\right)$, headaches, vomiting and diarrhoea, which was diagnosed as malaria and treated successfully with chloroquine. On 15 March 1989, he experienced rapidly progressive dysarthria and an unsteadiness on walking. A week later, the patient was referred to our hospital.

On admission, he was afebrile, but appeared chronically ill and complained of severe fatigue. There was a recent history of weight loss. Cardio-pulmonary and abdominal examinations were normal. There was no rash or lymphadenopathy. Neurological examination revealed a cerebellar syndrome interfering with a normal gait, and a less severe bilateral cerebellar ataxia. There was no abnormality of the cranial nerves, neither was there any nystagmus, or motor or sensory deficit. Tendon reflexes were present bilaterally, but were more prominent on the right side.

The following laboratory studies were normal: complete blood count, erythrocyte sedimentation rate, electrolytes, glucose, blood urea nitrogen, liver function tests, electrophoresis of plasma proteins and amylase. Serological tests for HIV1, HIV2, syphilis, Epstein Barr virus, cytomegalovirus, hepatitis B surface antigen, herpes simplex virus 1 were negative. A radiograph of the thorax, and the cerebral CT scan and MRI were normal. Blood and urine cultures were negative. The CSF was clear, under normal pressure, with six lymphocytes, and a normal glucose concentration. Protein was $0.7 \mathrm{~g} / \mathrm{L}$ and the gamma globulin count $18 \%$ with polyclonal banding. Bacteriological studies of the CSF were negative. The EEG showed diffuse slow waves, suggesting an encephalopathy.

During the following days, while there was no fever, the patient became icteric, and developed a hepatosplenomegaly confirmed by echography. There was also a pan- cytopenia. Peripheral blood films were positive for plasmodium parasites and an indirect immunofluorescent test was positive at $1 / 2560$, suggesting falciparum species. Treatment by mefloquine produced a rapid and complete recovery of the hepatosplenomegaly, pancytopenia and icterus. The cerebellar ataxia improved at the same time. The gait returned to normal two months later. Subsequent blood films for plasmodium were negative.

Even though the so called "cerebellar syndrome" is a well known but uncommon clinical presentation of malaria, ' there are few reported cases in the neurological literature. Lemercier $e t a l^{2}$ found a transient cerebellar syndrome in two of three patients presenting with severe febrile attacks, but they were usually less prominent than the other general and neurological signs of the attack. Nevertheless, the authors insisted on the frequency of the lesions involving the cerebellum, or its connections on neuropathological examination. Our patient had some common features with the 12 cases from Sri Lanka reported by Senanayake: ${ }^{3}$ cerebellar ataxia, sometimes associated with nystagmus, occurring in a febrile patient during the 15 days following a febrile attack of falciparum malaria. Gametocytes were present in the blood of these patients and the cerebellar signs subsided one to three months after the antimalarial treatment was begun. In the 12 patients the delayed onset of the neurological deficit, the absence of general signs (splenomegaly was present in one patient) and the presence of gametocytes on repeated blood smears (four out of 12 patients) suggested an immuno-allergic mechanism rather than a direct toxic event due to the plasmodium, even though there was a complete neurological recovery.

Our patient had different clinical features from those of the cases reported from Sri Lanka, but were similar to those reported by Girard et $a l^{4}$ and Garin et al. ${ }^{5}$ Clinical and laboratory findings in our patient make a viral or toxic cause unlikely.

P CHAINE F WOIMANT ORABIANU $M$ HAGUENAU Department of Neurology, Hôpital Lariboisiere, Hopital Pariboisiere,
75010 Paris, France

1 Castellani A, Chalmers AJ. Manual of tropical medicine, 2nd ed. London: Bailliere Tindall and Cox, 1913:885.

2 Lemercier G, Bert J, Nouhouayi A, et al. Coll. Le neuropaludisme: aspects électro-encépLe neuropaludisme: aspects electro-encep-
halographiques, neuro-pathologiques, halographiques, neuro-pathologiques,
problèmes physiopathologiques. Path Biol problèmes phys

3 Senanayake I. Delayed cerebellar ataxia: a new complication of falciparum malaria? $B M J$ 1987;294:1253-4

4 Girard PM, de Broucker T, Fryer D, et al. Cerebellar syndrome in mild Plasmodium falciparum malaria. Trans Roy Soc Trop Med Hygiene 1988;82:204.

5 Garin JP, Monier P, Mojon M, et al. Coll. Syndrome cérébelleux au cours d'un Girard-Lyon-S.P.C.M. Editions, 1976.

\section{A case of childhood Kufs' disease}

Kufs' disease is a form of neuronal ceroidlipofuscinosis (NCL), characterised by progressive epilepsy and dementia with motor 
disturbances and the accumulation in the neurons of abnormal lipopigments with fingerprint or granular ultrastructural pat, terns. Kufs' disease differs from the other forms of NCL by the absence of retinal degeneration and by a later age of onset. However, cases with similar features and course have been reported in children. ${ }^{1-4}$

Our case fulfils criteria for Kufs' disease and is remarkable by its early onset and by the predominance of psychiatric features.

A nine year old boy was the only child of boy's previous medical history was unremarkable except for splenectomy at the age of four years six months for traumatic rupture. Development had proceeded normally.

At the age of five he developed hyperkinetic behaviour and temporo-spatial disorientation. His school performance had been good through the first grades but later deteriorated rapidly. He was referred at the age of nine for assessment of intellectual deterioration.

Psychological testing revealed a full scale IQ of 42 (WISC R), IQ had been found to be 89 (Terman Merril) at the age of five. He had sudden frequent falls with no ataxia or epileptic seizures. His gestures were slow and aimless. Coordination of fine motor movements was poor. Neurological examination was otherwise unremarkable. He was indifferent to surroundings, had echolalia and communication was impossible. CSF, electrophysiological studies: electroencephalogram (EEG), electromyogram, electroretinogram and CT scan were normal. Vacuolated lymphocytes were not found. Serum long chain fatty acids were normal. Light and electron microscopy of biopsy samples of the skin and liver showed numerous osmiophilic bodies in smooth muscle cells which were considered nonspecific.

He continued to deteriorate and at the age of 14 he was bedridden, cachectic, stuporous, seizures often triggered by nursing. He died one year later.

EEG at the age of 11 showed slowing of background activity at $8 \mathrm{~Hz}$ with fast sharp activity in the occipital area, and spontaneous generalised spike and slow wave discharges. healthy, non-consanguineous parents. The with marked spasticity and intractable

Two years later intermittent photic stimulation elicited paroxysmal spikes over the posterior areas, each spike being synchronous with the flash for frequencies of 3-20 Hz (fig 1). Lower frequencies were not tested. Repeat CT scan showed generalised atrophy.

Other examinations remained normal including normal electroretinogram and visual evoked responses, normal nerve conduction velocities and normal lysosomal enzyme activities in leucocytes. Hexosaminidase A was tested with specific sulphated synthetic substrate. Somatosensory evoked potentials and urinary dolichol studies were not carried out.

Diagnosis was established by rectal biopsy at the age of 14 . On semithin sections, dense granules appeared in the cytoplasm of neurons of the Auerbach plexus. These deposits were autofluorescent. By electron microscopy, the osmiophilic granules were packed with fingerprint profiles. There were also a few cytosomes with lamellar profiles. There was no evidence of storage in other cells (fig 2).

Symptoms were limited to dementia and behavioural changes over a period of more than five years suggesting psychiatric disease. Seizures and pyramidal signs developed later when he was 11 . All laboratory examinations were unhelpful including skin biopsies at ages nine and 11 .

EEG sensitivity to photic stimulation of the type seen in this patient is very unusual. It has been already reported in several cases ${ }^{57-8}$ and is likely to be of diagnostic significance. The patients of Berkovic et $a l^{5}$ were also photosensitive at frequencies of 1 to $100 \mathrm{~Hz}$.

Although no necropsy was performed, we considered that the finding of specific inclusions in neuronal cells in the rectal plexus by electron microscopy confirms the diagnosis. Dom et $a l^{9}$ have also found lipopigmentary granules ballooning the nerve cells in Auerbach's plexus in an adult case confirmed at necropsy, and Berkovic ${ }^{5}$ reported dense granules in neurons of Meissner's plexus in his first case. Because rectal biopsy contains neurons it appears superior to muscle or conjunctival biopsy which may be negative. ${ }^{56}$ Fingerprintprofiles arenot completely specific for NCL; abnormal lipopigment deposits of

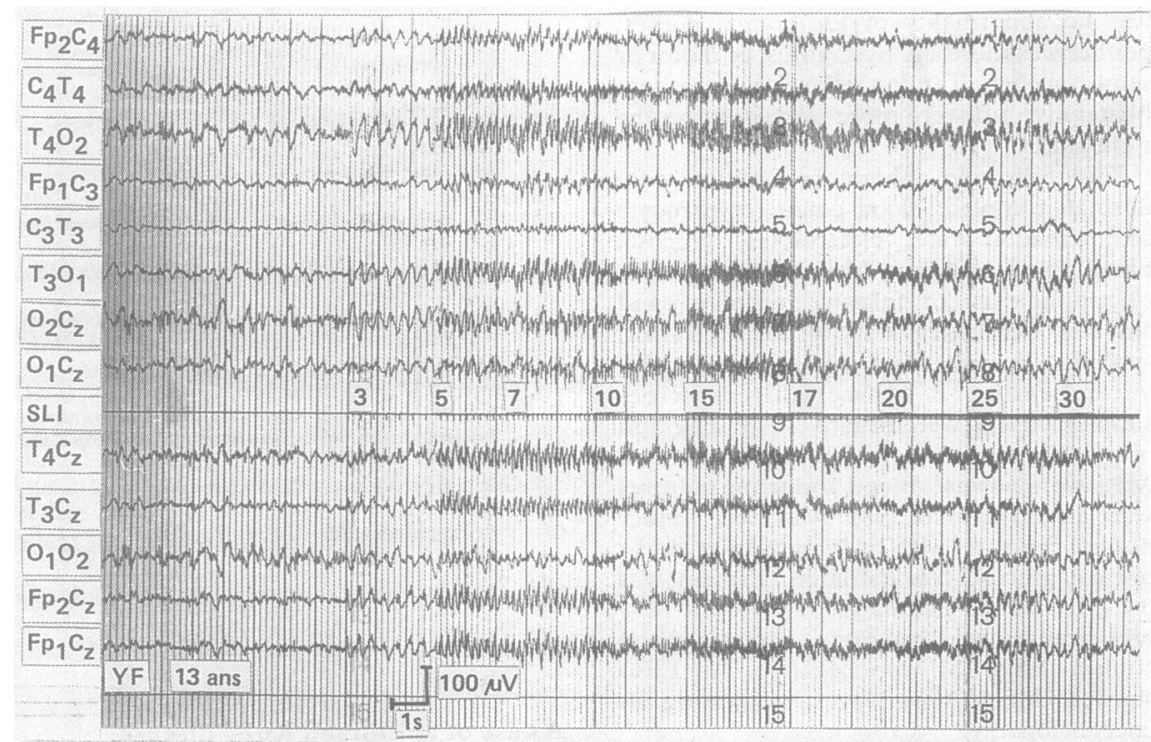

Figure 1 Abnormal background activity consisted mainly of slow waves and fast sharp activity in occipital areas with spontaneous epileptiform discharges. Intermittent photic stimulation at 3-20 hz elicited a marked photoparoxysmal response even at flash frequencies of $3 \mathrm{hz} . \mathrm{At} 5 \mathrm{hz}$, typical synchronised spike and wave discharges.

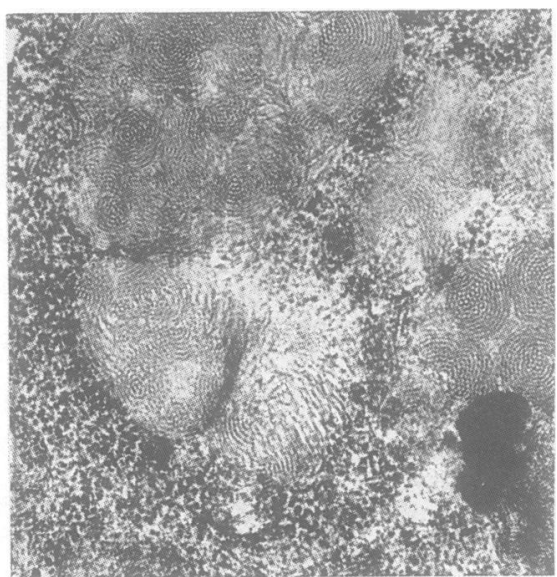

Figure 2 Rectal biopsy: electron micrograph showing fingerprint profiles in osmiophilic complex inclusions in a neuron of Auerbach's plexus $(17000 \times 2)$.

Kufs' disease may be difficult to distinguish from lipofuscin that accumulates in normal ageing or from the deposit of other storage diseases especially the late forms of GM2 gangliosidosis. The diagnosis, however, seems reasonably certain if abundant fingerprint profiles made of systems of paired parallel lines straight or curving accumulate in the neurons.

Despite the early onset of the disease in our case (five years), the lack of retinal disorder excludes infantile or juvenile types and is consistent with Kufs' disease. ${ }^{6}$ According to Berkovic et $a l^{5}$ the diagnosis of Kufs' disease was confirmed on the basis of pathological data in 50 published cases out of a total of 118 cases reviewed. The mean age of clinical onset was $29 \cdot 7$ years. The youngest patient was 11 years. ${ }^{1}$ Eight further patients from three different families were not accepted by Berkovic et $a l^{5}$ as cases of Kufs' disease because they occurred in childhood and no electron microscopic examination was available. ${ }^{2-4}$ These eight patients, however, had progressive dementia with no visual failure and were similar in all respects to adult Kufs' disease. The age of onset ranged from one to six years in one family, and from three to 10 in another. The abnormal storage seen on light microscopy was consistent with this diagnosis. Ultrastructural studies of cases such as these and ours are essential for diagnosis and may establish a link between childhood diseases with a Kufs'-like presentation and classical adult or adolescent onset Kufs' disease.

We thank Dr Aicardi for his advice.

$$
\begin{array}{r}
\text { MA BARTHEZ-CARPENTIER } \\
\text { C BILLARD } \\
\text { J MAHEUT } \\
\text { JJ SANTINI } \\
\text { Unité de Neurologie-Neurochirurgie Infantile, } \\
\text { Hôpital Clocheville, Tours } \\
\text { MM RUCHOUX } \\
\text { Département de Neuropathologie, Hôpital } \\
\text { Bretonneau, Tours, France }
\end{array}
$$

1 Greenwood RS, Nelson JS. Atypical neuronal ceroid-lipofuscinosis. Neurology 1978, 28:710-7.

2 Walter FK. Uber familiäre Idiote. Zeitschrift für die Gesamte Neurologie und Psychiatrie 1918, 40:349-84.

3 Jervis GA. Familial idiocy due to neuronal J Psychiatry 1950;107:409-14. 
4 Wakutani $K$, Nakamura $H$, Fukuda $M$, Inagak $\mathrm{T}$, Makihara S. An autopsy case of VogtSpielmeyer type of cerebral lipidosis. Clinical Neurology (Tokyo) 1967;7:81-7.

5 Berkovic SF, Carpenter S, Andermann F, Andermann E, Wolfe LS. Kufs' disease: critical reappraisal. Brain 1988;111:27-62.

6 Libert J, Martin JJ, Ceuterick C. Protracted and atypical forms of ceroid-lipofuscinosis. In: Armstrong D, Koppang N, Rider JA, eds. Ceroid-lipofuscinosis (Batten's disease) Amsterdam: Elsevier, 1982:42-59.

7 Pallis CA, Duckett S, Pearse AGE. Diffuse lipofuscinosis of the central nervous system Neurology (Minneapolis) 1967;17:381-94.

8 Vercruyssen A, Martin JJ, Ceuterick C, Jakobs Kercruyssen A, Martin JJ, Ceuterick C, Jakobs diagnostic value of biopsies and of neurophysiological investigations. $J$ Neurol Neurosurg Psychiatry 1982;45:1056-9.

9 Dom R, Brucher JM, Ceuterick C, Carton H, Martin JJ. Adult ceroid-lipofuscinosis (Kufs disease) in two brothers. Retinal and viscera storage in one; diagnostic muscle biopsy in the other. Acta Neuropathologica (Berlin) 1979, 45:67-72.

\section{Hemiageusia: an unusual presentation} of multiple sclerosis

Patients with multiple sclerosis (MS) rarely complain of taste disturbances, although electrogustatory examinations often demonstrate dysfunction of the taste pathway in patients with advanced disease, especially in those with prominent brainstem involvement.

A 25 year old native American man presented with a two day history of gradually progressive loss of taste on the entire right half of his tongue (hemiageusia). One week later he developed numbness of the right inner cheek, double vision, and a tendency to fall to the left. He had clockwise rotatory nystagmus, right internuclear ophthalmoplegia, left central facial palsy, left hyperreflexia, and intention tremor with the right hand. The right hemiageusia was unchanged. The gag reflex was diminished on the right side, and the right palate and the right inner cheek were numb. Routine laboratory tests were all normal. CSF analysis revealed no red cells, five lymphocytes, $53 \mathrm{mg} / \mathrm{dL}$ protein, normal glucose, $5.6 \mathrm{ng} /$ $\mathrm{mL}$ myelin basic protein (normal range $0-5 \cdot 1$ $\mathrm{ng} / \mathrm{mL}$ ), and three oligoclonal bands without correlates in serum.

Eleven days after the onset of the taste disturbance he developed paroxysms of pain around the right eye, perioral numbness and tingling sensation in the right cheek. New

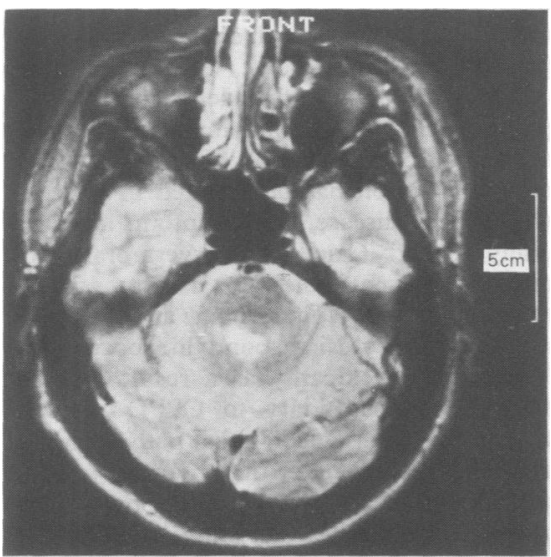

Figure T2-weighted axial MRI scan (1.5 tesla). Increased signal noted by the floor of the fourth ventricle. findings were a decreased right corneal reflex and hypaesthesia to pain and temperature in the distribution of the right mandibular nerve. Cranial MRI demonstrated multiple, bilateral, periventricular areas of increased T2 signal. A similar lesion was found in the right medulla on the floor of the fourth ventricle (fig). In the CSF myelin basic protein was $16.2 \mathrm{ng} / \mathrm{mL}$ and five oligoclona bands without serum correlate were identified. The patient was treated with a 10 day course of $1 \mathrm{gm} /$ day intravenous methylprednisone followed by a two week oral prednisone taper, without improvement.

Thirteen months after presentation he developed acute left hemiparesis. MRI demonstrated an increase in the number of periventricular lesions of increased T2 signal and a new right sided lesion at the expected location of the internal capsule. Visual evoked potentials and lower extremity somatosensory evoked potentials showed prolonged wave latencies. CSF showed an elevated protein $(63 \mathrm{mg} / \mathrm{dL})$ and myelin basic protein $(14.8 \mathrm{mg} / \mathrm{mL})$, and five oligoclonal bands. The patient was treated with a 10 day course of IV methylprednisone and his hemiparesis recovered. On examination he still had the previously described deficits.

Hemiageusia involving the entire right half of the tongue is usually explained by a lesion in the ipsilateral nucleus solitarius, where fibres from the lingual nerve (anterior two thirds of the tongue) and fibres from the glossopharyngeal nerve (posterior third) come together. ${ }^{2}$ Recent evidence suggests the presence of an accessory taste pathway through the trigeminal nerve. ${ }^{3}$ Hypogeusia is found in $5-10 \%$ of patients with advanced $M S^{1}$ and is frequently associated with sensory involvement of the trigeminal nerve. ${ }^{3-5}$ Taste disturbance as the initial symptom of MS has previously been reported only by $\mathrm{Harris}{ }^{\circ} \mathrm{His}$ patient, a 21 year old woman, developed numbness of the right side of her face and right sided hemiageusia, that persisted for one year. Four years later she presented with trigeminal neuralgia, but it was not until 10 years later, that multifocal symptoms led to the diagnosis of $\mathrm{MS} .^{6}$ The remarkable aspect of our case is that right-sided hemiageusia was the sole presenting symptom, although the investigation demonstrated multifocal central nervous system lesions. Right trigeminal sensory involvement occurred almost two weeks after the hemiageusia, and prominent, more widespread brainstem symptomatology developed only later. ALVARO PASCUAL-LEONE IRFAN ALTAFULLAH ANIL DHUNA Department of Neurology, University of Minnesota and Hennepin County Medical Center, Minneapolis, MN, USA

1 Rollin H. Geschmackstörungen bei multipler Sklerose. Laryng Rhinol 1976;55:678.

2 Carpenter MB. Human neuroanatomy. 7th ed. Baltimore: Williams and Wilkins, 1976.

3 Grant R, Ferguson MM, Strang R, Turners JW, Bone I. Evoked taste thresholds in a normal population and the application of electrogustometry to trigeminal nerve disease. $J$ Neurol Neurosurg Psychiat 1987;50:12.

4 Spillane JD, Wells CEC. Isolated trigeminal neuropathy. Brain 1959;82:391.

5 Lecky BRF, Hughes RAC, Murray NMF. Trigeminal sensory neuropathy: a study of 22 cases. Brain 1987;110:1463.

6 Harris W. Rare forms of paroxysmal trigeminal neuralgia, and their relation to disseminated neuralgia, and their relation
sclerosis. BMJ 1950;2:1015.
Paroxysmal kinesigenic choreoathetosis as presenting symptom of multiple sclerosis

Paroxysmal kinesigenic choreoathetosis (PKC) is characterised by attacks of uni- or bilateral choreoathetosis precipitated by sudden or fast movements. The acquired form of symptomatic PKC may be a manifestation of underlying structural or metabolic disease. ${ }^{\prime}$ PKC has previously been reported in eight patients as the first symptom of multiple sclerosis. ${ }^{2}$ We describe a patient with PKC as the presenting symptom of multiple sclerosis, in whom the lesions were localised by MRI.

A 35 year old female lawyer noticed diplopia and had minor attacks of sudden halts with her right foot when she started walking as if she had "stepped into glue". She noticed that the attacks were also precipitated by a sudden noise or the unexpected appearance of a cyclist or a car. She first sought medical attention five months later when she developed urgency of micturition and a diminution of dexterity of her right hand. The attacks now included slurred speech and rotational posturing of the head, right arm and leg lasting a few seconds and occurring five to 10 times daily. The attacks were provoked by emotion, acceleration of movement, speaking and writing. Neurological examination was normal. Two months later she was occasionally able to avert the attacks by completely arresting her movements as soon as prodromal symptoms occurred. The attacks occurred now five to 10 times per hour and lasted from about five to fifty seconds.

Neurological examination revealed internuclear ophthalmoplegia, cerebellar gait, dystonia of the right leg and hypertonic hemiparesis of the right side. Cerebrospinal fluid contained 26 mononuclear leukocytes, a slightly increased protein content with an increased IgG level and non-specific multiple oligoclonal bands in the alkaline region at isoelectric focussing. CT scan of the head showed a paraventricular hypodense area in the region of the caudate nucleus. MRI revealed in the proton density images and the $\mathrm{T}_{2}$ weighted pictures high signal emitting lesions paraventricular, in the putamen and

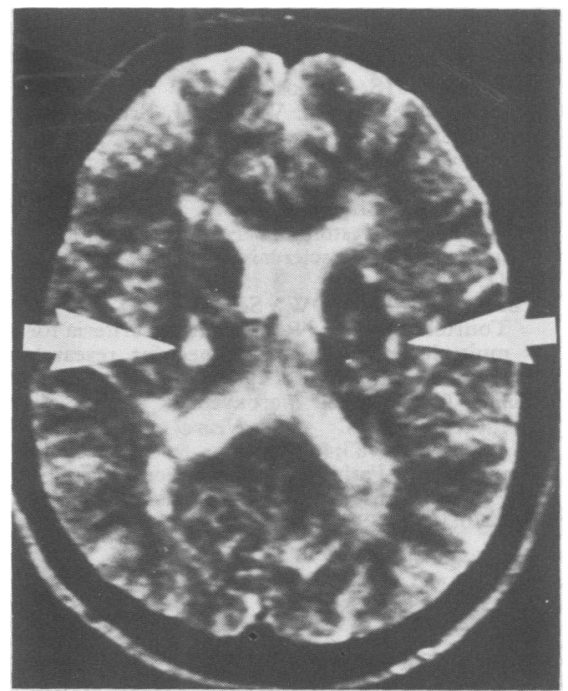

Figure Transverse section through the lateral ventricle with $T_{2}$ weighted images of the MRI demonstrates high signal emissions in the right globus pallidus and thalamus and in the subcortical paraventricular regions in both hemispheres. 